\title{
Regularized Channel Inversion for Simultaneous Confidential Broadcasting and Power Transfer: A Large System Analysis
}

\author{
Biao He* Member, IEEE, Nan Yang, Member, IEEE, \\ Shihao Yan, Member, IEEE, and Xiangyun Zhou, Member, IEEE
}

\begin{abstract}
We propose for the first time new transmission schemes based on linear precoding to enable simultaneous confidential broadcasting and power transfer (SCBPT) in a multiuser multi-input single-output (MISO) network, where a BS with $N$ antennas simultaneously transmits power and confidential messages to $K$ single-antenna users. We first design two transmission schemes based on the rules of regularized channel inversion (RCI) for both power splitting (PS) and time switching (TS) receiver architectures, namely, RCI-PS and RCI-TS schemes. For each scheme, we derive channel-independent expressions to approximate the secrecy sum rate and the harvested power in the large-system regime where $K, N \rightarrow \infty$ with a fixed ratio $\beta=K / N$. Based on the large-system results, we jointly optimize the regularization parameter of the RCI and the PS ratio or the TS ratio such that the secrecy sum rate is maximized subject to an energy-harvesting constraint. We then present the tradeoff between the secrecy sum rate and the harvested power achieved by each scheme, and find that neither scheme always outperforms the other one. Motivated by this fact, we design an RCI-hybrid scheme based on the RCI and a newly proposed hybrid receiver architecture. The hybrid receiver architecture takes advantages of both the PS and TS receiver architectures. We show that the RCI-hybrid scheme outperforms both the RCI-PS and RCI-TS schemes.
\end{abstract}

Index Terms-Physical layer security, confidential broadcasting, simultaneous wireless information and power transfer (SWIPT), linear precoding.

\section{INTRODUCTION}

$\mathbf{E}$ NERGY harvesting from the environment has been widely recognized as a promising candidate to provide perpetual power supplies and prolong the lifetime of wireless networks with low cost and high convenience. Spurred by Grover and Sahai's pioneer work [1], simultaneous wireless information and power transfer (SWIPT) has recently sparked a significant amount of attention. This is due to its ability of transporting both energy that is collected at the energy harvester and information that is decoded by the information decoder at the same time, through radio frequency (RF) signals from the base station (BS). It is highlighted that SWIPT is particularly suitable for the scenario where wireline charging

B. He is with the Department of Electronic and Computer Engineering, The Hong Kong University of Science and Technology, Hong Kong (email: eebiaohe@ust.hk).

N. Yang, S. Yan, and X. Zhou are with the Research School of Engineering, The Australian National University, Canberra, ACT 2601, Australia (e-mail: \{nan.yang, shihao.yan, xiangyun.zhou\}@anu.edu.au).

This work was supported by the Australian Research Council under Discovery Project Grant DP150103905. (Corresponding author: B. He.) and battery replacement are hazardous or infeasible. Recently developed receiver architectures for SWIPT include power splitting (PS) and time switching (TS) [2,3]. The receiver with PS uses a signal splitter to allocate the power between information decoding and energy harvesting. Differently, the receiver with TS implements a switcher to allocate the time between information decoding and energy harvesting.

Security becomes a crucial issue when SWIPT is adopted in a multiuser broadcasting channel. In such a channel, the transmitted information is received at not only the intended receiver but also the power receivers and/or potential eavesdroppers. Traditionally, cryptographic technologies are used to ensure wireless communication secrecy. However, techniques based on the encryption may not be suitable for SWIPT, since the required high computational capability will lead to high energy consumption and a burden on power transfer. As an effective alternative, physical layer security has been recently investigated to tackle the security issue for SWIPT, e.g., [4-9]. Physical layer security [10-13] provides secure data communications by exploiting the characteristics of wireless channels, such as interference, fading, and noise. Motivated by its great potential, physical layer security for SWIPT has been studied in different scenarios, e.g., multipleinput single-output (MISO) network with a single intended receiver $[4,5]$, relay networks $[6,7]$, cooperative networks [8], and multicast networks [9].

Although [4-9] explored the design of physical layer security with SWIPT, the secrecy for SWIPT in multi-antenna broadcast networks aiming at achieving confidential broadcasting has not been touched in the aforementioned literature. Differing from the wiretap channel, confidential broadcasting enables multiple messages to be securely transmitted to multiple users in the network. Specifically, each message is intended for one of the users but needs to be kept secret from the others. Spurred by its great importance, confidential broadcasting has been elaborately studied in conventional wireless networks without the consideration of power transfer, e.g., [14-20]. We note that the introduction of confidential broadcasting into SWIPT has not been thoroughly studied in the literature. The challenge brought by this introduction is to properly address the interference signals in SWIPT, which can also be used for wireless power transfer. To tackle this challenge, it is a must to understand the tradeoff between secrecy and energy in the problem of simultaneously confidential broadcasting and power transfer (SCBPT) and to carefully control and utilize 
the interference amongst multiple users. It is found that the optimal beamforming design for SCBPT was studied in [21], with the objective of minimizing the transmit power subject to secrecy-rate and energy-harvesting constraints. However, the tradeoff between secrecy and energy was not presented in [21] and only the PS receiver architecture was considered.

In this work, we comprehensively investigate the problem of SCBPT. Specifically, we design new transmission schemes as per the rules of linear precoding for two practical receiver architectures, i.e., PS and TS. We consider a single-cell network where an $N$-antenna BS simultaneously transmits power and independent confidential messages to $K$ single-antenna users. The BS adopts linear precoding based on the regularized channel inversion (RCI) [22] to achieve SCBPT. Moreover, we propose a new hybrid receiver architecture to reap the joint benefits of both PS and TS. The primary contributions of this paper are summarized as follows.

1) We design transmission schemes based on RCI precoding with both PS and TS receiver architectures, namely, RCI-PS and RCI-TS schemes. For each scheme, the precoding matrix is designed to tradeoff the received signal power, the information leakage, and the interference via a regularization parameter. The tradeoff between confidential information transmission and wireless power transfer is further regulated by the PS ratio in RCI-PS scheme or the TS ratio in RCI-TS scheme.

2) We derive new channel-independent expressions for the secrecy sum rate and the harvested power achieved by the designed schemes in the large-system regime, where $K, N \rightarrow \infty$ with a fixed ratio $\beta=K / N$. The large-system expressions do not depend on the channel realizations, which eliminates the huge computational burden of performance evaluation incurred by Monte Carlo simulations. Numerical results are provided to confirm the accuracy of our large-system expressions, even for finite $K$ and $N$.

3) We optimize the design of transmission schemes based on the large-system expressions. We jointly optimize the regularization parameter of the RCI and the PS ratio or the TS ratio, aiming at maximizing the secrecy sum rate subject to an energy-harvesting constraint. We present and compare the tradeoff between the secrecy sum rate and the harvested power for each scheme. An important conclusion is reached that neither the RCI-PS scheme nor the RCI-TS scheme always outperforms the other.

4) We design an RCI-hybrid scheme based on the RCI with a newly proposed hybrid receiver architecture. We derive large-system expressions for the secrecy sum rate and the harvested power achieved by the RCI-hybrid scheme. We further optimize the design of RCI-hybrid scheme based on the large-system expressions. We show that the RCI-hybrid scheme outperforms both the RCI-PS and RCI-TS schemes in terms of the achieved tradeoff between the secrecy sum rate and the harvested power.

It is worth mentioning that the analysis of the proposed schemes in this work relies on the assumption of Gaussian input signals for information-theoretic optimality. We note that practical signals are often of a finite alphabet and nonGaussian. Thus, investigating secure transmissions with finitealphabet inputs is of practical importance, e.g., [23], and will be considered as future work.

Throughout the paper, we adopt the following notations: $(\cdot)^{T}$ and $(\cdot)^{H}$ denote the transpose and conjugate transpose of a vector or a matrix, respectively, $\operatorname{Tr}(\cdot)$ denotes the trace of a matrix, $\|\cdot\|$ denotes the Euclidean norm of a vector, $\mathbb{E}\{\cdot\}$ denotes the expectation operation, $[x]^{+}=\max (x, 0)$, $\stackrel{a . s}{\longrightarrow}$ and $\stackrel{\text { i.p. }}{\longrightarrow}$ denote almost sure convergence and convergence in probability, respectively.

\section{NETWORK MODEL}

We study SCBPT in a multiuser MISO broadcast network consisting of $K$ single-antenna users and one $N$-antenna BS. The received signal at user $k$ is given by [24-26]

$$
y_{k}=\sqrt{\gamma_{k}} \mathbf{h}_{k} \mathbf{x}+n_{k},
$$

where $\gamma_{k}$ denotes the distance-dependent path gain, the $1 \times N$ row vector $\mathbf{h}_{k}$ denotes the channel state vector, $\mathbf{x}$ denotes the transmitted data vector, and $n_{k} \sim \mathcal{C N}\left(0, \sigma_{n}^{2}\right)$ denotes the narrowband additive white Gaussian noise (AWGN) introduced by the receive antenna. The path gain captures the attenuation of the signal waves with distance and is equivalent to the path-loss coefficient used in some other papers. In this work, we assume that all users have the same path gain such that $\gamma_{k}=\gamma, \forall k$. We assume that the antennas at the BS and the users are sufficiently spaced apart such that all links between transmit and receive antennas are uncorrelated. The small-scale fading effects between the BS and the users are modeled as independent and identically distributed (i.i.d.) complex Gaussian variables with zero mean and unit variance, i.e., $\mathbf{h}_{k} \sim \mathcal{C N}\left(\mathbf{0}, \mathbf{I}_{N}\right)$. For the sake of brevity, we denote $\mathbf{H}=\left[\mathbf{h}_{1}^{H} \mathbf{h}_{2}^{H} \cdots \mathbf{h}_{K}^{H}\right]^{H}$ as the channel state matrix between the BS and all users.

In addition, we assume that each user $k$ perfectly knows $\mathbf{h}_{k}$ and feeds it back to the BS. Finally, we assume that the BS perfectly recovers the CSI from feedback information. We note that perfect CSI is assumed to be available in this work. If channel estimation errors exist, the achievable secrecy rates of the proposed schemes would decrease. As such, the achievable secrecy rate derived in this work can be regarded as an upper bound on the achievable secrecy rate for the network with channel estimation errors.

\section{A. Confidential Broadcasting}

The first requirement in the design of SCBPT is confidential broadcasting. To meet this requirement, the message for each user $k$ needs to be securely transmitted such that the unintended users obtain zero information. Since the behavior of users cannot be fully controlled by the BS in designing secure transmission strategies, we consider a worst-case scenario in the design of SCBPT such that all other $K-1$ users are treated as potential eavesdroppers. In this scenario, we assume that the eavesdroppers can jointly eavesdrop on the message in a collaborative manner. Therefore, the alliance of $K-1$ cooperating eavesdroppers is equivalent to a single 
eavesdropper with $K-1$ distributed receive antennas, which is denoted by eavesdropper $\tilde{k}$. The cooperating eavesdroppers decode their own signals and share them with each other. It follows that the cooperating eavesdroppers are able to perform interference cancellation, leaving only the signal for the intended user. Note that the consideration of the worst-case scenario is necessary in designing confidential broadcasting networks; see, e.g., [16-19].

The secrecy performance of the network is measured by the secrecy sum rate, denoted by $R_{s}$, which is mathematically formulated as

$$
R_{s}=\sum_{k=1}^{K} R_{k}
$$

where $R_{k}$ denotes the perfect secrecy rate at user $k$. Specifically, $R_{k}$ quantifies the instantaneous rate at which user $k$ can receive the message confidentially and reliably.

\section{B. Wireless Power Transfer}

The second requirement in the design of SCBPT is wireless power transfer. To meet this requirement, the BS transmits wireless power to users for maintaining their receiver operation. The wireless power transfer brings great convenience for the energy access to users in the network where interconnecting wires are inconvenient, hazardous, or impossible. The harvested power at user $k$ needs to be larger than a threshold, $\Gamma_{k}$, which is the minimum power to maintain the receiver operation at user $k$ [27]. In this work, we assume that all users are the same type of receiver device, such that the energyharvesting constraint is the same for all users, i.e., $\Gamma_{k}=\Gamma, \forall k$.

\section{RCI Precoding}

We consider that the RCI [22] is adopted at the BS to achieve confidential broadcasting and power transfer. As a linear precoder, the $\mathrm{RCI}^{1}$ has a low signal processing complexity and a full ability of controlling the information leakage and interference amongst multiple users $[17,19]$. As per the rules of the RCI, the precoding vector for the message for user $k$ is given by

$$
\mathbf{w}_{k}=c\left(\mathbf{H}^{H} \mathbf{H}+\alpha \mathbf{I}_{N}\right)^{-1} \mathbf{h}_{k}^{H},
$$

where $c$ is the scaling factor to ensure the transmit power constraint at the $\mathrm{BS}$ and $\alpha$ is the real non-negative regularization parameter. Note that the scaling factor $c$ is identical for all $s_{k}$ to ensure that the power constraint is satisfied, as per the rules of the RCI precoding [22]. Such a structure of the RCI with identical $c$ for all messages has been widely adopted in the literature, e.g., $[17,19,20,22,28]$. We clarify that $\alpha$ achieves a tradeoff between the signal power at the receiver and the crosstalk amongst users. In particular, the RCI with $\alpha \rightarrow \infty$ converges to conjugate beamforming, which maximizes the intended received signal power. In addition, the RCI with $\alpha \rightarrow 0$ converges to zero forcing, which minimizes the crosstalk amongst users.

\footnotetext{
${ }^{1}$ We note that RCI is named as the regularized zero forcing in some existing studies.
}

The transmitted data vector $\mathbf{x}$ is given by

$$
\mathbf{x}=\sum_{k=1}^{K} \mathbf{w}_{k} s_{k},
$$

where $s_{k}$ denotes the message for user $k$. We assume that the messages for different users are independent. We also impose a unit average power constraint on $s_{k}$ such that $\mathbb{E}\left\{\mathbf{s s}^{H}\right\}=$

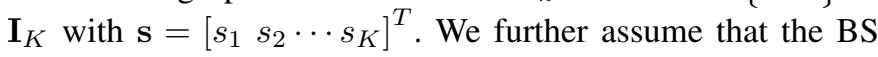
is subject to an average transmit power constraint given by $\mathbb{E}\left\{\|\mathbf{x}\|^{2}\right\}=P$. Then, the scaling factor $c$ is written as

$$
c=\sqrt{\frac{P}{\operatorname{Tr}\left(\left(\mathbf{H}^{H} \mathbf{H}+\alpha \mathbf{I}_{N}\right)^{-2} \mathbf{H}^{H} \mathbf{H}\right)}} .
$$

The problem of designing the RCI precoder is to find the regularization parameter $\alpha$ in order to obtain the scaling factor $c$ in (5) and the precoding vector $\mathbf{w}_{k}$ in (3).

It is worth mentioning that the RCI precoder is not the optimal linear precoder. The optimal linear precoder usually does not have any closed-form expression, and the determination of the optimal linear precoding is a complicated and timeconsuming optimization process. As previously mentioned, the RCI achieves a balance between the conjugate beamforming and the zero forcing with low complexity, although suboptimal. Thus, a great amount of research attention has been paid to the RCI-based design in the literature, e.g., $[16,17,19,20$, $22,28,29]$, focusing on different scenarios. We highlight that this paper is the first study on the RCI-based transmission schemes for the SCBPT.

\section{RCI-PS SCHEME AND RCI-TS SCHEME}

In this section, we present the RCI-PS and RCI-TS schemes for SCBPT. For each scheme, we first analytically characterize the secrecy sum rate and the harvested power as functions of channel realizations. We then derive new expressions, which are independent of channel realizations for the ease of computation, to characterize the secrecy sum rate and the harvested power in the large-system regime. In this regime, both the number of users, $K$, and the number of transmit antennas at the BS, $N$, approach infinity with a fixed ratio $\beta=K / N$. Finally, based on the large-system results, we devise the optimal scheme design for maximizing the secrecy sum rate subject to the energy-harvesting constraint.

\section{A. RCI-PS Scheme}

The principle of the RCI-PS scheme is illustrated as Figure 1(a). For the received signal power of $P_{k}$ at user $k$, a fraction $0 \leq \rho_{k} \leq 1$ of the power, i.e., $\rho_{k} P_{k}$, is used for information transmission. The remaining power, $\left(1-\rho_{k}\right) P_{k}$, is used for energy harvesting. We refer to $\rho_{k}$ as the PS ratio. The choice of the PS ratio $\rho_{k}$ directly affects the transmission rate and the harvested power. Note that in practice the PS is achieved by a RF signal splitter which allocates power between information decoding and energy harvesting. As such, the PS ratio is determined by the structure of the RF signal splitter at the user. In this work, we consider a low-complexity 


\begin{tabular}{|c|}
\hline $\begin{array}{c}\text { Information Transmission } \\
\left(\rho_{k} P_{k}\right)\end{array}$ \\
\hline $\begin{array}{c}\text { Energy Harvesting } \\
\left(\left(1-\rho_{k}\right) P_{k}\right)\end{array}$ \\
\hline
\end{tabular}

(a) Key parameters in the RCI-PS scheme.

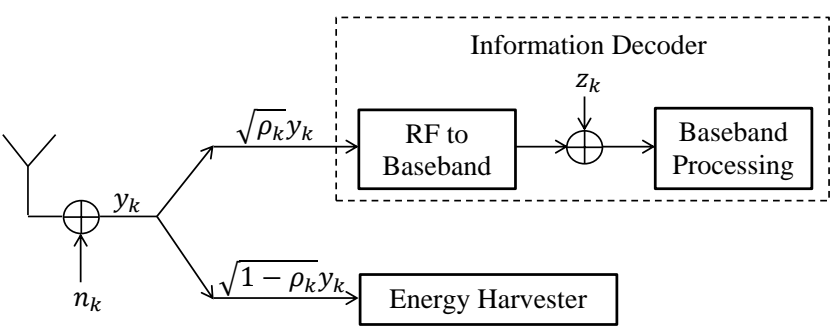

(b) Block diagram of the receiver in the RCI-PS scheme.

Fig. 1: Illustration of the key parameters and the block diagram of the receiver in the RCI-PS scheme.

and practical structure of the signal splitter such that the PS ratio cannot be arbitrarily changed once it is implemented, and the PS ratio is designed offline $[3,30]$. We note that some theoretical studies ideally assumed that the PS ratio can be arbitrarily changed, such advanced design with high complexity is too difficult to implement in conventional signal splitters [31].

1) Information Transfer and Power Transfer: The block diagram of the receiver at user $k$ with PS architecture is shown as Figure 1(b). The original RF signal received at user $k$ is denoted by $y_{k}$. The narrowband AWGN introduced by the receive antenna is denoted by $n_{k} \sim \mathcal{C N}\left(0, \sigma_{n}^{2}\right)$.

For the information transfer, the power splitter at user $k$ splits a fraction $\rho_{k}$ of the received signal power to the information decoder. We assume that all users are the same type of receiver device, and hence, the PS ratio is the same for all users, i.e., $\rho_{k}=\rho, \forall k$. The received signal at the information decoder of user $k$ after down conversion is given by

$$
y_{k}^{\mathrm{ID}}=\sqrt{\rho} y_{k}+z_{k}=\sqrt{\rho}\left(\sqrt{\gamma} \mathbf{h}_{k} \mathbf{x}+n_{k}\right)+z_{k}
$$

where $z_{k} \sim \mathcal{C N}\left(0, \sigma_{z}^{2}\right)$ denotes the sampled AWGN due to the RF band to baseband signal conversion. The received signal vector at the information decoders of all users is given by

$$
\mathbf{y}^{\mathrm{ID}}=\sqrt{\rho}(\sqrt{\gamma} \mathbf{H} \mathbf{x}+\mathbf{n})+\mathbf{z},
$$

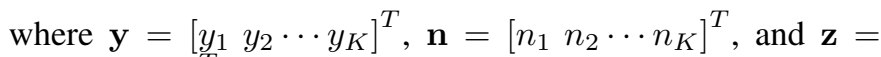
$\left[\begin{array}{llll}z_{1} & z_{2} & \cdots & z_{K}\end{array}\right]^{T}$. Using RCI, the transmitted data vector $\mathbf{x}$ is given by (4), and $y_{k}^{\mathrm{ID}}$ is accordingly rewritten as

$$
\begin{aligned}
y_{k}^{\mathrm{ID}}= & \sqrt{\rho \gamma} c \mathbf{h}_{k}\left(\mathbf{H}^{H} \mathbf{H}+\alpha \mathbf{I}_{N}\right)^{-1} \mathbf{h}_{k}^{H} s_{k} \\
& +\sqrt{\rho \gamma} c \mathbf{h}_{k}\left(\mathbf{H}^{H} \mathbf{H}+\alpha \mathbf{I}_{N}\right)^{-1} \mathbf{H}_{\tilde{k}}^{H} \mathbf{s}_{\tilde{k}}+\sqrt{\rho} n_{k}+z_{k},
\end{aligned}
$$

where $\mathbf{H}_{\tilde{k}}$ and $\mathbf{s}_{\tilde{k}}$ are obtained from $\mathbf{H}$ and $\mathbf{s}$ by removing the row corresponding to user $k$, respectively. The received signal vector at the information decoder of eavesdropper $\tilde{k}$ after down conversion is written as

$$
\mathbf{y}_{\tilde{k}}^{\mathrm{ID}}=\sqrt{\rho \gamma} c \mathbf{H}_{\tilde{k}}\left(\mathbf{H}^{H} \mathbf{H}+\alpha \mathbf{I}_{N}\right)^{-1} \mathbf{h}_{k}^{H} s_{k}+\sqrt{\rho} \mathbf{n}_{\tilde{k}}+\mathbf{z}_{\tilde{k}},
$$

where $\mathbf{y}_{\tilde{k}}, \mathbf{n}_{\tilde{k}}$, and $\mathbf{z}_{\tilde{k}}$ are obtained from $\mathbf{y}, \mathbf{n}$, and $\mathbf{z}$ by removing the row corresponding to user $k$, respectively. As mentioned before, we assume that the PS ratio is designed offline, and cannot be arbitrarily changed once it is implemented. It is worth mentioning that the eavesdropper can increase the signal power at the information decoder if it is able to change the PS ratio and sets its $\rho$ as 1 . However, we clarify that assuming $\rho=1$ at the eavesdropper is still impractical even if the PS ratio can be changed. In this work, we consider that the wireline charging is infeasible at users, and the operational power at the user is obtained only from the BS by wireless power transfer. The harvested power at the user needs to be larger than a threshold for maintaining the receiver operation. If the potential eavesdropper splits all of the received signal to the information decoder by setting its $\rho=1$, the received power at the energy harvester would not be enough for maintaining the operation of receiving signals. Then, the eavesdropper cannot obtain the information.

Then, the SINRs for the message $s_{k}$ at the information decoder of intended user $k$ and the information decoder of eavesdropper $\tilde{k}$ are given by

$$
\operatorname{SINR}_{k}=\frac{\rho \gamma c^{2}\left|\mathbf{h}_{k}\left(\mathbf{H}^{H} \mathbf{H}+\alpha \mathbf{I}_{N}\right)^{-1} \mathbf{h}_{k}^{H}\right|^{2}}{\rho \gamma c^{2} \psi+\rho \sigma_{n}^{2}+\sigma_{z}^{2}}
$$

and

$$
\operatorname{SINR}_{\tilde{k}}=\frac{\rho \gamma c^{2}\left|\mathbf{H}_{\tilde{k}}\left(\mathbf{H}^{H} \mathbf{H}+\alpha \mathbf{I}_{N}\right)^{-1} \mathbf{h}_{k}^{H}\right|^{2}}{\rho \sigma_{n}^{2}+\sigma_{z}^{2}},
$$

respectively, where

$$
\psi=\mathbf{h}_{k}\left(\mathbf{H}^{H} \mathbf{H}+\alpha \mathbf{I}_{N}\right)^{-1} \mathbf{H}_{\tilde{k}}^{H} \mathbf{H}_{\tilde{k}}\left(\mathbf{H}^{H} \mathbf{H}+\alpha \mathbf{I}_{N}\right)^{-1} \mathbf{h}_{k}^{H} .
$$

Note that the interference vector $\mathbf{s}_{\tilde{k}}$ does not appear in (9) and the received interference signal power does not appear in (11). These are due to the worst-case consideration of cooperative eavesdroppers who can collaberatively perform the interference cancellation. As such, the secrecy sum rate achieved in the RCI-PS scheme is given by

$$
R_{s, \mathrm{PS}}=\sum_{k=1}^{K}\left[\log _{2}\left(\frac{1+\frac{\rho \gamma c^{2}\left|\mathbf{h}_{k}\left(\mathbf{H}^{H} \mathbf{H}+\alpha \mathbf{I}_{N}\right)^{-1} \mathbf{h}_{k}^{H}\right|^{2}}{\rho \gamma c^{2} \psi+\rho \sigma_{n}^{2}+\sigma_{z}^{2}}}{1+\frac{\rho \gamma c^{2}\left|\mathbf{H}_{\tilde{k}}\left(\mathbf{H}^{H} \mathbf{H}+\alpha \mathbf{I}_{N}\right)^{-1} \mathbf{h}_{k}^{H}\right|^{2}}{\rho \sigma_{n}^{2}+\sigma_{z}^{2}}}\right)\right]^{+} .
$$

For the power transfer, the power splitter at user $k$ splits a fraction $1-\rho_{k}$ of the received signal power to the energy harvester. With $\rho_{k}=\rho, \forall k$, the received signal at the energy harvester of user $k$ is given by

$$
y_{k}^{\mathrm{EH}}=\sqrt{1-\rho}\left(\sqrt{\gamma} \mathbf{h}_{k}\left(\mathbf{H}^{H} \mathbf{H}+\alpha \mathbf{I}_{N}\right)^{-1} \mathbf{H}^{H} \mathbf{s}+n_{k}\right) .
$$

In this work, we assume that the harvested energy due to the noise $n_{k}$ is very small and thus ignored [2]. Then, the harvested power at user $k$ in the RCI-PS scheme is given by

$$
E_{k, \mathrm{PS}}=\epsilon(1-\rho) \gamma c^{2}\left(\left\|\mathbf{h}_{k}\left(\mathbf{H}^{H} \mathbf{H}+\alpha \mathbf{I}_{N}\right)^{-1} \mathbf{H}^{H}\right\|^{2}\right),
$$


where $0<\epsilon \leq 1$ denotes the energy conversion efficiency at the energy harvester.

2) Large-System Analysis: It is evident that the secrecy sum rate in (13) and the harvested power in (15) depend on the realization of each channel $\mathbf{h}_{k}$. This means that the performance evaluation based on (13) and (15) can only be performed through time-consuming numerical simulations. In order to reduce the complexity of performance evaluation, we resort to the large-system analysis to produce channel-independent expressions that explicitly characterize the secrecy sum rate and the harvested power. Such analysis is performed in the large-system regime where $K$ and $N$ approach infinity with a fixed ratio, $\beta=K / N$.

In the following Theorem 1, we present the large-system results for the RCI-PS scheme.

Theorem 1: For the RCI-PS scheme, the large-system secrecy sum rate and the large-system harvested power convergence in probability to deterministic quantities given by (16) and (17), respectively, where $\phi=\alpha / N$ and $g(\beta, \phi)$ is the solution of $x$ to $x=\left(\phi+\frac{\beta}{1+x}\right)^{-1}$.

Proof: See Appendix A.

Corollary 1: For any given PS ratio $\rho$, the large-system harvested power at the user, $E_{k, \mathrm{PS}}^{\infty}$, increases as the regularization parameter $\alpha$ increases. The maximum harvested power is obtained when $\alpha \rightarrow \infty$, given by

$$
\lim _{\alpha \rightarrow \infty} E_{k, \mathrm{PS}}^{\infty}=\epsilon(1-\rho) \gamma P\left(1+\frac{1}{\beta}\right) .
$$

Proof: See Appendix B.

Notably, the RCI with $\alpha \rightarrow \infty$ converges to the conjugate beamforming. Thus, Corollary 1 implies that it is wise to simply adopt the conjugate beamforming at the BS if only power is transmitted.

3) Optimal Design: We now optimize the design of the RCI-PS scheme based on the large-system results. We jointly optimize the regularization parameter $\alpha$ and the PS ratio $\rho$ that maximize the large-system secrecy sum rate subject to the energy-harvesting constraint. We note that both $\alpha$ and $\rho$ play pivotal roles in determining the network performance. Specifically, $\alpha$ handles the tradeoff between the signal power at the intended receiver and the cross-talk amongst users, while $\rho$ manages the tradeoff between the information transfer and the power transfer.

Problem Formulation: The problem of optimizing the design of the RCI-PS scheme is formulated as:

$$
\begin{array}{cl}
\max _{\alpha, \rho} & R_{s, \mathrm{PS}}^{\infty}, \\
\text { s.t. } & E_{k, \mathrm{PS}}^{\infty} \geq \Gamma, \alpha \geq 0,0 \leq \rho \leq 1 .
\end{array}
$$

Feasible Constraint: We first derive the feasible energyharvesting constraint subject to which a positive secrecy rate can be obtained. The feasible range of energy-harvesting constraint is the range of harvested power that can be achieved. Thus, the maximum feasible energy-harvesting constraint is equal to the maximum achievable harvested power. From (17) and Corollary 1, we find that the maximum achievable harvested power is obtained when $\alpha \rightarrow \infty$ and $\rho \rightarrow 0$.
Thus, the feasible energy-harvesting constraint for the RCIPS scheme is given by

$$
\Gamma \leq E_{k, \mathrm{PS}}^{\infty}(\alpha \rightarrow \infty, \rho \rightarrow 0)=\epsilon \gamma P\left(1+\frac{1}{\beta}\right) .
$$

Optimal Solution: The optimal solution of $\alpha$, denoted by $\alpha_{\mathrm{PS}}^{*}$, is given by

$$
\alpha_{\mathrm{PS}}^{*}=f_{\alpha}\left(\rho=\rho_{\mathrm{PS}}^{*}\right),
$$

where $\rho_{\mathrm{PS}}^{*}$ denotes the optimal solution of $\rho$. The function $f_{\alpha}(\rho)$ in (22) is defined by

$$
f_{\alpha}(\rho)=\max \left(\alpha_{o}, \alpha_{l}\right),
$$

where

$\alpha_{o}=\left[\frac{3 \theta_{1}+\theta_{1}^{2}-(\beta-1)^{2}-\left(\theta_{1}+\beta-1\right) \sqrt{\theta_{1}^{2}+(\beta+2) \theta_{1}+(\beta-1)^{2}}}{3\left(\theta_{1}+\beta+2\right) / N}\right]^{+}$

and

$$
\alpha_{l}= \begin{cases}0, & \text { if } \epsilon(1-\rho) \gamma P\left|\frac{1}{\beta}-1\right|>\Gamma \\ N q, & \text { otherwise, }\end{cases}
$$

with $\theta_{1}=\beta\left(\rho \sigma_{n}^{2}+\sigma_{z}^{2}\right) /(\rho \gamma P)$ and $q$ is the solution of $\phi$ to

$$
\frac{\epsilon(1-\rho) \gamma P(\beta+\phi g(\beta, \phi)(1+g(\beta, \phi)))}{\beta(1+g(\beta, \phi))}=\Gamma .
$$

The optimal solution of $\rho$, denoted by $\rho_{\mathrm{PS}}^{*}$, is obtained by numerically solving the problem:

$$
\begin{array}{cl}
\max _{\rho} & R_{s, \mathrm{PS}}^{\infty}\left(\rho, \alpha=f_{\alpha}(\rho)\right), \\
\text { s.t. } & 0<\rho \leq 1-\frac{\Gamma \beta}{\epsilon \gamma P(1+\beta)} .
\end{array}
$$

In the following, we provide the proof of the proposed optimal solution for the RCI-PS scheme.

Proof: We first derive the first derivative of $R_{s, \mathrm{PS}}^{\infty}$, given by (16), with respect to $\alpha$. With $\phi=\frac{\alpha}{N}$, we have $\frac{\partial R_{s, \mathrm{PS}}^{\infty}}{\partial \alpha}=$ $\frac{1}{N} \frac{\partial R_{s, \mathrm{PS}}^{\infty}}{\partial \phi}$. Given $g(\beta, \phi)=\left(\phi+\frac{\beta}{1+g(\beta, \phi)}\right)^{-1}$, we find that $\phi=\frac{1+g(\beta, \phi)-\beta g(\beta, \phi)}{g(\beta, \phi)(1+g(\beta, \phi))}$ and $\frac{\partial g(\beta, \phi)}{\partial \phi}=\frac{-g(\beta, \phi)(1+g(\beta, \phi))^{2}}{\beta+\phi(1+g(\beta, \phi))^{2}}$. We then obtain

$$
\begin{aligned}
& \frac{\partial R_{s, \mathrm{PS}}^{\infty}}{\partial \phi}=\frac{\partial R_{s, \mathrm{PS}}^{\infty}}{\partial g(\beta, \phi)} \cdot \frac{\partial g(\beta, \phi)}{\partial \phi} \\
& =\frac{2 g(\beta, \phi)^{2} \omega^{4} \nu\left(2 \omega \nu-\beta\left(g(\beta, \phi)^{2}-\nu+2 \nu g(\beta, \phi)-1\right)\right)}{\beta((\beta g(\beta, \phi)-g(\beta, \phi)-2) g(\beta, \phi)-1)\left(\omega^{2}+\nu\right)^{3}}
\end{aligned}
$$

where $\nu=\frac{\rho \gamma P}{\rho \sigma_{n}^{2}+\sigma_{z}^{2}}$ and $\omega=1+g(\beta, \phi)$. We find that there are two possibilities for the sign of $\partial R_{s, \mathrm{PS}}^{\infty} / \partial \alpha$ when $\alpha \geq 0$ : 1) $\partial R_{s, \mathrm{PS}}^{\infty} / \partial \alpha$ is always negative or 2) $\partial R_{s, \mathrm{PS}}^{\infty} / \partial \alpha$ is positive for small $\alpha$ and becomes negative as $\alpha$ increases. Thus, for a given $\rho$, the value of $\alpha$ that maximizes $R_{s, \mathrm{TS}}^{\infty}$ is equal to either zero or a unique positive value. Then, we obtain the optimal $\alpha$ without considering the energy-harvesting constraint as $\alpha_{o}$ in (24) by seeking the solution of $\alpha$ to $\partial R_{s, \mathrm{PS}}^{\infty} / \partial \alpha=0$. With the energy-harvesting constraint $E_{k, \mathrm{PS}}^{\infty} \geq \Gamma$, we find that there is a lower bound on $\alpha$ for a given $\rho$, since $E_{k, \mathrm{PS}}^{\infty}$ is a monotonously increasing function of $\alpha$. We next obtain the lower bound on $\alpha$ for a given $\rho$ as $\alpha_{l}$ in (25) by solving for $\alpha$ in the equation $E_{k, \mathrm{PS}}^{\infty}=\Gamma$. Therefore, the optimal $\alpha$ for a given $\rho$ is given 


$$
R_{s, \mathrm{PS}}^{\infty}= \begin{cases}K\left[\log _{2}\left(\frac{1+\frac{\rho \gamma P g(\beta, \phi)\left(1+\frac{\phi}{\beta}(1+g(\beta, \phi))^{2}\right)}{\rho \gamma P+\left(\rho \sigma_{n}^{2}+\sigma_{z}^{2}\right)(1+g(\beta, \phi))^{2}}}{1+\frac{\rho \gamma P}{\left(\rho \sigma_{n}^{2}+\sigma_{z}^{2}\right)(1+g(\beta, \phi))^{2}}}\right)\right]^{+}, & \text {if } \alpha>0 \\ K \log _{2}\left(1+\frac{\rho \gamma P(1-\beta)}{\left(\rho \sigma_{n}^{2}+\sigma_{z}^{2}\right) \beta}\right), & \text { if } \alpha=0 \text { and } \beta \leq 1 \\ K\left[\log _{2}\left(\frac{\beta^{4}+\frac{\rho \gamma P \beta^{3}(\beta-1)}{\rho \sigma_{n}^{2}+\sigma_{z}^{2}}}{\left(\beta^{2}+\frac{\rho \gamma P(\beta-1)^{2}}{\rho \sigma_{n}^{2}+\sigma_{z}^{2}}\right)^{2}}\right)\right]^{+}, & \text {if } \alpha=0 \text { and } \beta>1 .\end{cases}
$$

$$
E_{k, \mathrm{PS}}^{\infty}= \begin{cases}\frac{\epsilon(1-\rho) \gamma P(\beta+\phi g(\beta, \phi)(1+g(\beta, \phi)))}{\beta(1+g(\beta, \phi))}, & \text { if } \alpha \neq 0 \\ \epsilon(1-\rho) \gamma P \frac{1-\beta}{\beta}, & \text { if } \alpha=0 \text { and } \beta \leq 1 \\ \epsilon(1-\rho) \gamma P \frac{\beta-1}{\beta}, & \text { if } \alpha=0 \text { and } \beta>1\end{cases}
$$

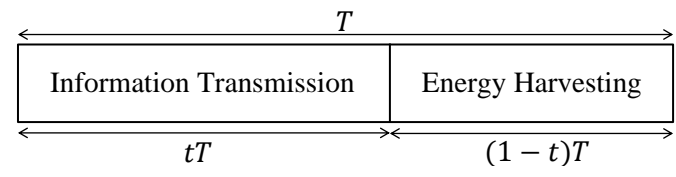

(a) Key parameters in the RCI-TS scheme.

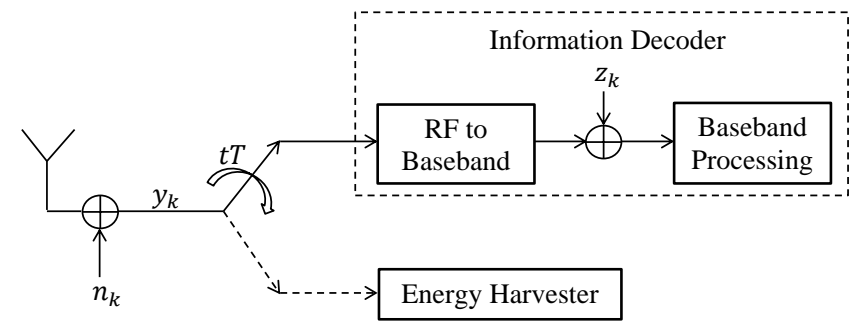

(b) Block diagram of the receiver in the RCI-TS scheme.

Fig. 2: Illustration of the key parameters and the block diagram of the receiver in the RCI-TS scheme.

by $f_{\alpha}(\rho)$ in (23). We note that the closed-form expression for $\rho_{\mathrm{PS}}^{*}$ is mathematically intractable. Hence, we determine $\rho_{\mathrm{PS}}^{*}$ by numerically solving the problem of (27). In addition, we find that the energy-harvesting constraint cannot be achieved if $\rho>1-\frac{\Gamma \beta}{\epsilon \gamma P(1+\beta)}$, and hence, the range to search of $\rho$ is given by (28). The way to numerically solve the problem (27) is a simple one dimensional search, and the details are given as follows. The lower and upper bounds on $\rho$ to search are given by (28). For each value of $\rho$, we first obtain the corresponding optimal $\alpha$ for the given $\rho$ by (23), and then get the maximum achievable $R_{s, \mathrm{PS}}^{\infty}$ for a given $\rho$. Finally, we compare the values of the maximum achievable $R_{s, \mathrm{PS}}^{\infty}$ for all given values of $\rho$, and find the optimal $\rho$ to the problem (27).

\section{B. RCI-TS Scheme}

The principle of the RCI-TS scheme is illustrated as Figure 2(a). In a block time of $T$, the BS spends a fraction $0 \leq t \leq 1$ of the block time, i.e., $t T$, for information transmission. The remaining block time, $(1-t) T$, is used for energy harvesting. We refer to $t$ as the TS ratio. The choice of the TS ratio $t$ directly affects the transmission rate and the harvested power.
1) Information Transfer and Power Transfer: The block diagram of the receiver at user $k$ with TS architecture is shown as Figure 2(b). During the period of information transfer (for $t T$ time), the RF signal $y_{k}$ received at user $k$ is sent to the information decoder. The received signal at the information decoder of user $k$ after down conversion is given by

$$
y_{k}^{\mathrm{ID}}=y_{k}+z_{k}=\sqrt{\gamma} \mathbf{h}_{k} \mathbf{x}+n_{k}+z_{k} .
$$

The received signal vector at the information decoders of all users is given by

$$
\mathbf{y}^{\mathrm{ID}}=\sqrt{\gamma} \mathbf{H} \mathbf{x}+\mathbf{n}+\mathbf{z} .
$$

With the RCI, the transmitted data vector $\mathbf{x}$ is given by (4), and $y_{k}^{\mathrm{ID}}$ is rewritten as

$$
\begin{aligned}
y_{k}^{\mathrm{ID}}= & \sqrt{\gamma} c \mathbf{h}_{k}\left(\mathbf{H}^{H} \mathbf{H}+\alpha \mathbf{I}_{N}\right)^{-1} \mathbf{h}_{k}^{H} s_{k} \\
& +\sqrt{\gamma} c \mathbf{h}_{k}\left(\mathbf{H}^{H} \mathbf{H}+\alpha \mathbf{I}_{N}\right)^{-1} \mathbf{H}_{\tilde{k}}^{H} \mathbf{s}_{\tilde{k}}+n_{k}+z_{k} .
\end{aligned}
$$

The received signal vector at the information decoder of eavesdropper $\tilde{k}$ after down conversion is given by

$$
\mathbf{y}_{\tilde{k}}=\sqrt{\gamma} c \mathbf{H}_{\tilde{k}}\left(\mathbf{H}^{H} \mathbf{H}+\alpha \mathbf{I}_{N}\right)^{-1} \mathbf{h}_{k}^{H} s_{k}+\mathbf{n}_{\tilde{k}}+\mathbf{z}_{\tilde{k}} .
$$

Then, the SINRs for the message $s_{k}$ at the information decoder of intended user $k$ and the information decoder of eavesdropper $\tilde{k}$ are given by

$$
\operatorname{SINR}_{k}=\frac{\gamma c^{2}\left|\mathbf{h}_{k}\left(\mathbf{H}^{H} \mathbf{H}+\alpha \mathbf{I}_{N}\right)^{-1} \mathbf{h}_{k}^{H}\right|^{2}}{\gamma c^{2} \psi+\sigma_{n}^{2}+\sigma_{z}^{2}}
$$

and

$$
\operatorname{SINR}_{\tilde{k}}=\frac{\gamma c^{2}\left|\mathbf{H}_{\tilde{k}}\left(\mathbf{H}^{H} \mathbf{H}+\alpha \mathbf{I}_{N}\right)^{-1} \mathbf{h}_{k}^{H}\right|^{2}}{\sigma_{n}^{2}+\sigma_{z}^{2}}
$$

respectively, where $\psi$ is defined in (12). Considering the TS ratio $t$ allocated for information transfer, the effective secrecy sum rate achieved in the RCI-TS scheme is given by

$$
R_{s, \mathrm{TS}}=\sum_{k=1}^{K}\left[t \log _{2}\left(\frac{1+\frac{\gamma c^{2}\left|\mathbf{h}_{k}\left(\mathbf{H}^{H} \mathbf{H}+\alpha \mathbf{I}_{N}\right)^{-1} \mathbf{h}_{k}^{H}\right|^{2}}{\gamma c^{2} \psi+\sigma_{n}^{2}+\sigma_{z}^{2}}}{1+\frac{\gamma c^{2}\left|\mathbf{H}_{\tilde{k}}\left(\mathbf{H}^{H} \mathbf{H}+\alpha \mathbf{I}_{N}\right)^{-1} \mathbf{h}_{k}^{H}\right|^{2}}{\sigma_{n}^{2}+\sigma_{z}^{2}}}\right)\right]^{+} .
$$

During the period of power transfer (for $(1-t) T$ time), the RF signal $y_{k}$ received at user $k$ is sent to the energy 
harvester. Note that during the period of power transfer, the BS does not transmit the useful (confidential) message, and the only objective is to maximize the received signal power at users. Thus, the BS simply applies the conjugate beamforming for power transfer, i.e., the RCI with $\alpha \rightarrow \infty$, which maximizes the received signal power at users. Using conjugate beamforming, the received signal at the energy harvester of user $k$ is given by

$$
y_{k}^{\mathrm{EH}}=\sqrt{\frac{\gamma P}{\operatorname{Tr}\left(\mathbf{H}^{H} \mathbf{H}\right)}} \mathbf{h}_{k} \mathbf{H}^{H} \mathbf{s}+n_{k},
$$

where the $\mathbf{s}$ for power transfer is independent with the confidential message to any users. Considering the time ratio $1-t$ allocated for power transfer, the effective harvested power in the RCI-TS scheme is given by

$$
E_{k, \mathrm{TS}}=\frac{(1-t) \epsilon \gamma P}{\operatorname{Tr}\left(\mathbf{H}^{H} \mathbf{H}\right)}\left\|\mathbf{h}_{k} \mathbf{H}^{H}\right\|^{2}
$$

2) Large-System Analysis: In the following, we derive channel-independent expressions for the secrecy sum rate and the harvested power in the large-system regime where $K \rightarrow \infty, N \rightarrow \infty$, and $\beta=K / N$. These expressions are presented in the following Proposition 1.

Proposition 1: For the RCI-TS scheme, the large-system secrecy sum rate and the large-system harvested power converge in probability to deterministic quantities given by (39) and

$$
E_{k, \mathrm{TS}}^{\infty}=(1-t) \epsilon \gamma P\left(1+\frac{1}{\beta}\right)
$$

respectively, where $\phi=\alpha / N$ and $g(\beta, \phi)$ is the solution of $x$ to $x=\left(\phi+\frac{\beta}{1+x}\right)^{-1}$.

Proof: Comparing (36) and (13), we find that $R_{s, \mathrm{TS}}=$ $t R_{s, \mathrm{PS}}(\rho=1)$. Comparing (38) and (15), we find that $E_{k, \mathrm{TS}}=$ $(1-t) \lim _{\alpha \rightarrow \infty} E_{k \text {,PS }}(\rho=0)$. Thus, the results for the RCITS scheme in Proposition 1 can be easily derived from the results for the RCI-PS scheme in Theorem 1, i.e., $R_{s, \text { TS }}^{\infty}=$ $t R_{s, \mathrm{PS}}^{\infty}(\rho=1)$ and $E_{k, \mathrm{TS}}^{\infty}=(1-t) \lim _{\alpha \rightarrow \infty} E_{k, \mathrm{PS}}^{\infty}(\rho=0)$.

3) Optimal Design: We now optimize the design of the RCI-TS scheme based on the large-system results. We determine the optimal regularization parameter $\alpha$ and the optimal TS ratio $t$ that maximize the large-system secrecy sum rate subject to the energy-harvesting constraint. We note that $\alpha$ handles the tradeoff between the signal power at the intended receiver and the cross-talk amongst users, whereas $t$ manages the tradeoff between the information transfer and the power transfer.

Problem Formulation: The problem of optimizing the design of the RCI-TS scheme is formulated as:

$$
\begin{aligned}
\max _{\alpha, t} & R_{s, \mathrm{TS}}^{\infty} \\
\text { s.t. } & E_{k, \mathrm{TS}}^{\infty} \geq \Gamma, \alpha \geq 0,0 \leq t \leq 1 .
\end{aligned}
$$

Feasible Constraint: We first derive the feasible energyharvesting constraint subject to which a positive secrecy rate can be obtained. The maximum achievable harvested power is obtained when $t \rightarrow 0$, and hence the feasible energy-harvesting constraint for the RCI-TS scheme is given by

$$
\Gamma<E_{k, \mathrm{TS}}^{\infty}(t \rightarrow 0)=\epsilon \gamma P\left(1+\frac{1}{\beta}\right),
$$

which is the same as the feasible energy-harvesting constraint for the RCI-PS scheme given in (21).

Optimal Solution: The optimal solutions of $\alpha$ and $t$, denoted by $\alpha_{\mathrm{TS}}^{*}$ and $t_{\mathrm{TS}}^{*}$, are given by

$$
\alpha_{\mathrm{TS}}^{*}=\left[\frac{3 \theta_{2}+\theta_{2}^{2}-(\beta-1)^{2}-\left(\theta_{2}+\beta-1\right) \sqrt{\theta_{2}^{2}+(\beta+2) \theta_{2}+(\beta-1)^{2}}}{3\left(\theta_{2}+\beta+2\right) / N}\right]_{(44)}^{+}
$$

and

$$
t_{\mathrm{TS}}^{*}=1-\frac{\Gamma \beta}{\epsilon \gamma P(1+\beta)},
$$

respectively, where $\theta_{2}=\beta\left(\sigma_{n}^{2}+\sigma_{z}^{2}\right) /(\gamma P)$.

In the following, we provide the proof of the proposed optimal solution for the RCI-TS scheme.

Proof: Since $R_{s, \mathrm{TS}}^{\infty}=t R_{s, \mathrm{PS}}^{\infty}(\rho=1)$, we can easily obtain $\alpha_{\mathrm{TS}}^{*}$ from (24) while having $\rho=1$. We find that $R_{s, \mathrm{TS}}^{\infty}$ in (39) is a monotonously increasing function of $t$. Then, the optimal $t$ is the maximum value of $t$ that satisfies the constraint of $E_{k, \mathrm{TS}}^{\infty}(t) \geq \Gamma$. We further find that $E_{k, \mathrm{TS}}^{\infty}$ in (40) is a monotonously decreasing function of $t$. Thus, we obtain the $t_{\mathrm{TS}}^{*}$ in (45) by solving for $t$ in the equation $E_{k, \mathrm{TS}}^{\infty}(t)=\Gamma$.

\section{Numerical Results}

In this subsection, we provide numerical results to examine the performance achieved by the proposed RCI-PS and RCITS schemes. For the numerical results in this paper, we set the parameters as $N=32, \beta=0.5, \epsilon=0.9, P=43 \mathrm{dBm}$, $\sigma_{n}^{2}=-70 \mathrm{dBm}$, and $\sigma_{z}^{2}=-50 \mathrm{dBm}$. The distance-dependent path gain $\gamma$ is modelled by [32] $\gamma=c_{0}\left(d / d_{0}\right)^{-m}$, where $c_{0}=-30 \mathrm{~dB}$ is the constant attenuation for the path loss at the reference distance $d_{0}=1 \mathrm{~m}, d=15 \mathrm{~m}$ is the distance between BS and users, and $m=2.7$ is the path-loss exponent. The unit of the large-system secrecy sum rate $R_{s}^{\infty}$ is bits per channel use (bpcu). The unit of the energy-harvesting constraint $\Gamma$ and the large-system harvested power $E_{k}^{\infty}$ is microwatt $(\mu \mathrm{W})$.

We first examine the accuracy of the large-system results. To compare the large-system secrecy sum rate, $R_{s}^{\infty}$, with the average secrecy sum rate in the network with finite $K$ and $N$, denoted by $\mathbb{E}\left\{R_{s}\right\}$, we introduce the normalized rate difference defined by

$$
\Delta R_{s}=\frac{\left|\mathbb{E}\left\{R_{s}\right\}-R_{s}^{\infty}\right|}{\mathbb{E}\left\{R_{s}\right\}},
$$

which quantifies the rate difference between $R_{s}^{\infty}$ and $\mathbb{E}\left\{R_{s}\right\}$ for finite $K$ and $N$. Similarly, to compare the large-system harvested power, $E_{k}^{\infty}$, with the average harvested power in the network with finite $K$ and $N$, denoted by $\mathbb{E}\left\{E_{k}\right\}$, we introduce the normalized power difference defined by

$$
\Delta E_{k}=\frac{\left|\mathbb{E}\left\{E_{k}\right\}-E_{k}^{\infty}\right|}{\mathbb{E}\left\{E_{k}\right\}},
$$

which quantifies the power difference between $E_{k}^{\infty}$ and $\mathbb{E}\left\{E_{k}\right\}$ for finite $K$ and $N$. 


$$
R_{s, \mathrm{TS}}^{\infty}= \begin{cases}K t\left[\log _{2}\left(\frac{1+\frac{\gamma P g(\beta, \phi)\left(1+\frac{\phi}{\beta}(1+g(\beta, \phi))^{2}\right)}{\gamma P+\left(\sigma_{n}^{2}+\sigma_{z}^{2}\right)(1+g(\beta, \phi))^{2}}}{1+\frac{\gamma\left(\sigma_{n}^{2}+\sigma_{z}^{2}\right)(1+g(\beta, \phi))^{2}}{2}}\right)\right]^{+}, & \text {if } \alpha>0 \\ K t \log _{2}\left(1+\frac{\gamma P(1-\beta)}{\left(\sigma_{n}^{2}+\sigma_{z}^{2}\right) \beta}\right), & \text { if } \alpha=0 \text { and } \beta \leq 1 \\ K t\left[\log _{2}\left(\frac{\beta^{4}+\frac{\gamma P \beta^{3}(\beta-1)}{\sigma_{n}^{2}+\sigma_{z}^{2}}}{\left(\beta^{2}+\frac{\gamma P(\beta-1)^{2}}{\sigma_{n}^{2}+\sigma_{z}^{2}}\right)^{2}}\right)\right]^{+}, & \text {if } \alpha=0 \text { and } \beta>1 .\end{cases}
$$

Table 1: Illustration of the accuracy of large-system approximations for RCI-PS scheme.

\begin{tabular}{|c||c|c|c|c|c|c|c|c|c|c|}
\hline$N$ & 8 & 12 & 16 & 20 & 24 & 28 & 32 & 36 & 40 & 44 \\
\hline$\Delta R_{s}$ & $15.3 \%$ & $6.5 \%$ & $3.2 \%$ & $1.8 \%$ & $0.99 \%$ & $0.67 \%$ & $0.44 \%$ & $0.31 \%$ & $0.23 \%$ & $0.18 \%$ \\
\hline$\Delta E_{k}$ & $6.6 \%$ & $3.5 \%$ & $2.2 \%$ & $1.5 \%$ & $1.0 \%$ & $0.87 \%$ & $0.65 \%$ & $0.51 \%$ & $0.25 \%$ & $0.14 \%$ \\
\hline
\end{tabular}

Table 2: Illustration of the accuracy of large-system approximations for RCI-TS scheme.

\begin{tabular}{|c||c|c|c|c|c|c|c|c|c|c|}
\hline$N$ & 8 & 12 & 16 & 20 & 24 & 28 & 32 & 36 & 40 & 44 \\
\hline$\Delta R_{s}$ & $16.9 \%$ & $7.5 \%$ & $3.8 \%$ & $2.1 \%$ & $1.0 \%$ & $0.77 \%$ & $0.54 \%$ & $0.37 \%$ & $0.28 \%$ & $0.20 \%$ \\
\hline$\Delta E_{k}$ & $3.6 \%$ & $1.4 \%$ & $0.80 \%$ & $0.50 \%$ & $0.46 \%$ & $0.36 \%$ & $0.33 \%$ & $0.14 \%$ & $0.10 \%$ & $0.08 \%$ \\
\hline
\end{tabular}

Tables 1 and 2 illustrate the accuracy of the large-system approximations over the size of network for the RCI-PS scheme and the RCI-TS scheme, respectively. We set $\rho=0.8$, $t=0.8$, and $\alpha=0.2$. We note that $\Delta R_{s}$ and $\Delta E_{k}$ decrease as $N$ increases in both tables. As shown in Table 1, both $\Delta R_{s}$ and $\Delta E_{k}$ are smaller than $1 \%$ when $N>24$. As shown in Table 2, both $\Delta R_{s}$ and $\Delta E_{k}$ are smaller than $1 \%$ when $N>24$. Based on the aforementioned observations, we find that the large-system analytical results exhibit good accuracy for finite $N$ and $K$ (e.g., $N \geq 24$ ). We also find that such accuracy improves when $N$ increases. We highlight that this finding does not contradict the assumption adopted in the large-system analysis. Indeed, such accuracy is an important advantage of the large-system analysis, which has been demonstrated in the literature, e.g, $[17,19,20,28,33]$. Furthermore, we notice that the large-system analytical results may not be directly applied to the cellular networks where the BS has a small number of antennas. However, we highlight that the largesystem analytical results are applicable in the (near) future networks. With the explosive demand for mobile data and the fast development of large-scale antenna array technologies, the scale of future networks is envisioned to be very large [34]. Experimental trials for a large-scale system where the BS has 64 transmit antennas have already been conducted [35]. Thus, our obtained results are applicable to future networks with a relatively large scale.

We now present the optimal design parameters for both the RCI-PS and RCI-TS schemes over the energy-harvesting constraint. Figure 3 plots the optimal regularization parameters $\alpha_{\mathrm{PS}}^{*}$ and $\alpha_{\mathrm{TS}}^{*}$ for the RCI-PS scheme and the RCI-TS scheme, respectively. As shown in the figure, $\alpha_{\mathrm{PS}}^{*}$ increases fast as $\Gamma$ increases. This is because that the RCI is adopt for both the information transfer and the power transfer in the RCIPS scheme. We need to increase $\alpha$ as the energy-harvesting constraint becomes stringent. In contrast, $\alpha_{\mathrm{TS}}^{*}$ keeps constant as $\Gamma$ increases. This is because that the RCI is adopt for only the information transfer in the RCI-TS scheme. The value of

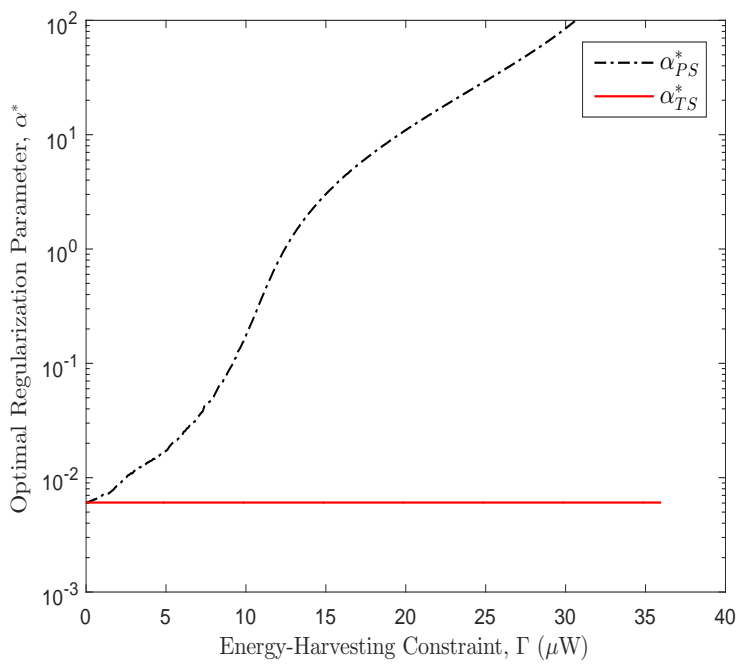

Fig. 3: The optimal regularization parameter versus the energyharvesting constraint.

$\alpha$ does not affect the performance of energy harvesting in the RCI-TS scheme. The value of $\alpha_{\mathrm{TS}}^{*}$ is independent with $\Gamma$, which can be seen from (44). Figure 4 plots the optimal PS ratio $\rho_{\mathrm{PS}}^{*}$ and the optimal TS ratio $t_{\mathrm{TS}}^{*}$ for the RCI-PS scheme and the RCI-TS scheme, respectively. As shown in the figure, both $\rho_{\mathrm{PS}}^{*}$ and $t_{\mathrm{TS}}^{*}$ decrease as $\Gamma$ increases, since we need to spend more power and/or time resources for the power transfer as the energy-harvesting constraint becomes stringent. We further note that $\rho_{\mathrm{PS}}^{*}$ decreases fast when $\Gamma$ is small, while $\rho_{\mathrm{PS}}^{*}$ decreases slowly when $\Gamma$ is large. In contrast, $t_{\mathrm{TS}}^{*}$ always decreases linearly as $\Gamma$ increases. Note that we have not claimed that the optimal design based on our largesystem analysis is optimal for small-size networks. Instead, our results show that the optimal design based on the largesystem analysis is near-optimal when the size of the network is sufficiently large, e.g., $N \geq 24$. This is because that the 


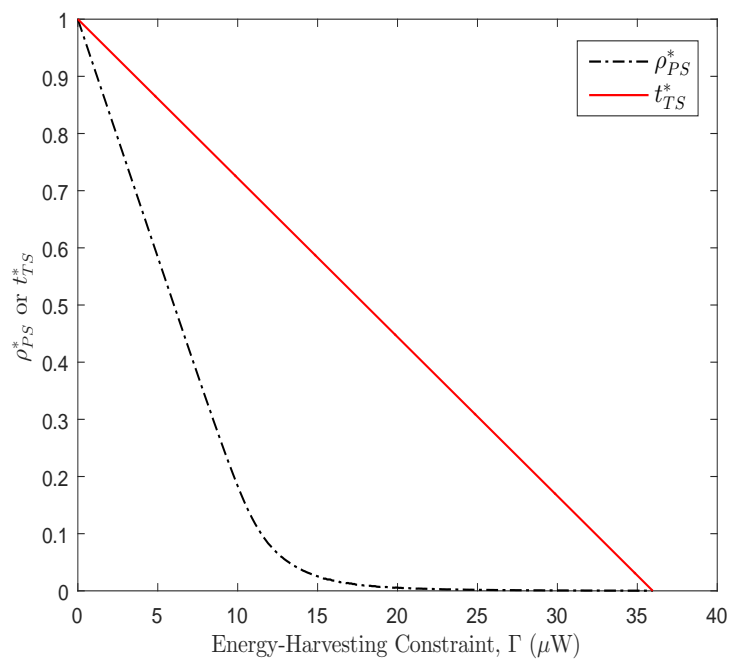

Fig. 4: The optimal PS ratio or TS ratio versus the energy-harvesting constraint.

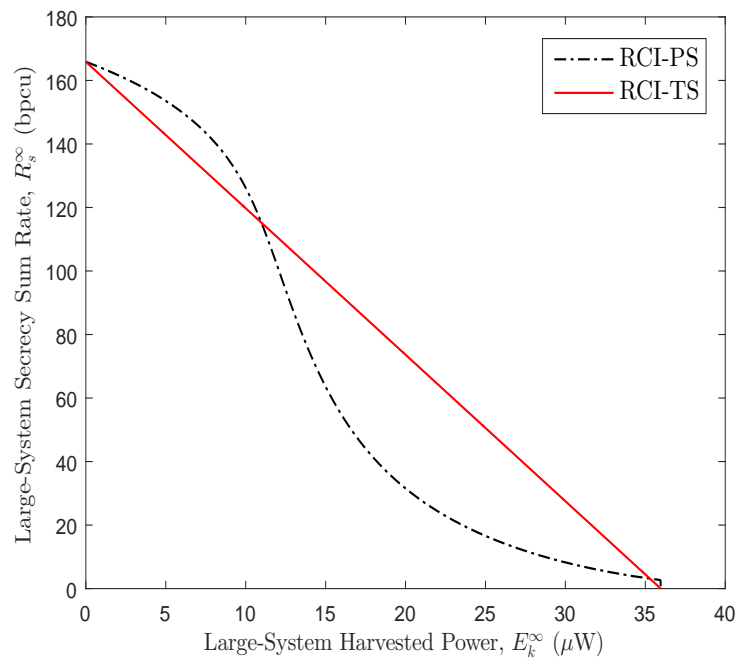

Fig. 5: The secrecy sum rate versus the harvested power.

objective function and the constraint, i.e., the secrecy sum rate and the harvested power, with $N \geq 24$, match precisely with the secrecy sum rate and the harvested power obtained from the large-system analysis. For small-size networks, the optimal design has to be performed using time-consuming simulations.

Finally, we demonstrate the tradeoff between the secrecy performance and the energy-harvesting performance achieved by the RCI-PS and RCI-TS schemes. Figure 5 plots $R_{s}^{\infty}$ versus $E_{k}^{\infty}$. Note that the area below the curve represents the achievable secrecy-energy region which consists of all achievable pairs of the secrecy sum rate and the harvested power. The $\left(R_{s}^{\infty}, E_{k}^{\infty}\right)$ pairs on the curve (i.e., the boundary of the secrecy-energy region) are obtained by the optimally designed scheme. As shown in the figure, to achieve better energy-harvesting performance, the secrecy performance of the system has to be scarified, and vice versa. Moreover, we find that the RCI-PS scheme sometimes outperforms the RCI-PS

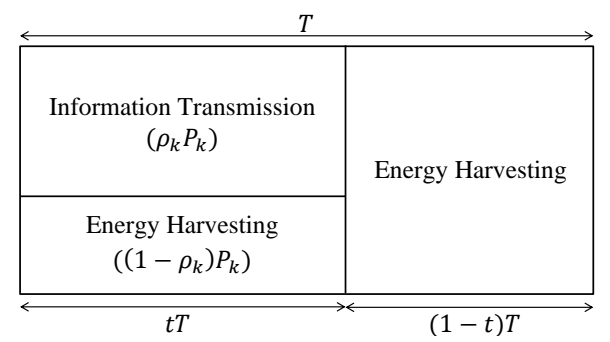

(a) Key parameters in the RCI-hybrid scheme.

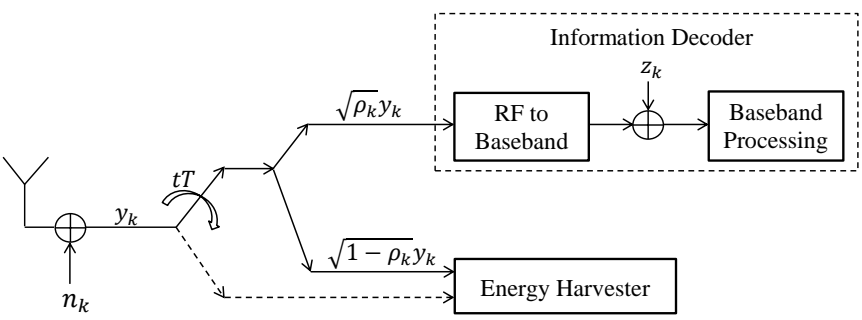

(b) Block diagram of the receiver in RCI-hybrid scheme.

Fig. 6: Illustration of the key parameters and the block diagram of the receiver in the RCI-hybrid scheme.

scheme and the RCI-TS scheme sometimes outperforms the RCI-PS scheme.

\section{RCI-HYBRID SCHEME}

As illustrated by Figure 5 in Section III, both RCI-PS and RCI-TS schemes have their own advantages, but neither of them always outperforms the other. This motivates us to propose an RCI-hybrid scheme in this section, which takes advantages of both schemes given in the previous section.

The principle of the RCI-hybrid scheme is illustrated as Figure 6(a). In a block time of $T$, the BS spends a fraction $0 \leq$ $t \leq 1$ of the block time, i.e., $t T$, for simultaneous information and power transfer. The remaining block time, $(1-t) T$, is used for only power transfer. During the period of simultaneous information and power transfer, the receiver of the user further splits the received signal. For the received signal power of $P_{k}$ at user $k$, a fraction $0 \leq \rho_{k} \leq 1$ of the power, i.e., $\rho_{k} P_{k}$, is used for information transmission, and the remaining power, $\left(1-\rho_{k}\right) P_{k}$, is used for energy harvesting.

\section{A. Information Transfer and Power Transfer}

The block diagram of the receiver at user $k$ with the hybrid architecture is shown as Figure 6(b). During the period of simultaneous information and power transfer (for $t T$ time), the power splitter at user $k$ splits a fraction $\rho_{k}$ of the received signal power to the information decoder and the remaining fraction $1-\rho_{k}$ of the received signal power to the energy harvester. Again, we assume that the PS ratio is the same for all users, i.e., $\rho_{k}=\rho, \forall k$. The BS adopts the RCI in this period, and the received signals at the information decoder and the energy harvested are the same as those given in the RCIPS scheme. The received signal at the information decode of user $k$, the received signal vector at the information decoder of eavesdropper $\tilde{k}$, and the received signal at the energy harvester 
of user $k$ are given by (8), (9), and (14), respectively. Then, from (13) with the consideration of the TS ratio $t$, we obtain the effective secrecy sum rate achieved in the RCI-hybrid scheme given by

$$
R_{s, \text { hybrid }}=\sum_{k=1}^{K}\left[t \log _{2}\left(\frac{1+\frac{\rho \gamma c^{2}\left|\mathbf{h}_{k}\left(\mathbf{H}^{H} \mathbf{H}+\alpha \mathbf{I}_{N}\right)^{-1} \mathbf{h}_{k}^{H}\right|^{2}}{\rho \gamma c^{2} \psi+\rho \sigma_{n}^{2}+\sigma_{z}^{2}}}{1+\frac{\rho \gamma c^{2}\left|\mathbf{H}_{\tilde{k}}\left(\mathbf{H}^{H} \mathbf{H}+\alpha \mathbf{I}_{N}\right)^{-1} \mathbf{h}_{k}^{H}\right|^{2}}{\rho \sigma_{n}^{2}+\sigma_{z}^{2}}}\right)\right]^{+}
$$

where $\psi$ is defined in (12). During the period of power transfer (for $(1-t) T$ time), the RF signal $y_{k}$ received at user $k$ is directly sent to the energy harvester. The BS applies the conjugate beamforming in this period. The received signal at the energy harvester of user $k$ in this period is the same as that in the RCI-TS scheme, i.e. (37). Then, from (15) and (38) with the consideration of the TS ratio, we obtain the harvested power at user $k$ in the RCI-hybrid scheme given by

$$
\begin{aligned}
E_{k, \text { hybrid }}= & t \epsilon(1-\rho) \gamma c^{2}\left\|\mathbf{h}_{k}\left(\mathbf{H}^{H} \mathbf{H}+\alpha \mathbf{I}_{N}\right)^{-1} \mathbf{H}^{H}\right\|^{2} \\
& +\frac{(1-t) \epsilon \gamma P}{\operatorname{Tr}\left(\mathbf{H}^{H} \mathbf{H}\right)}\left(\left\|\mathbf{h}_{k} \mathbf{H}^{H}\right\|^{2}\right)
\end{aligned}
$$

\section{B. Large-System Analysis}

We now derive channel-independent expressions for the secrecy sum rate and the harvested power in the large-system regime where $K \rightarrow \infty, N \rightarrow \infty$, and $\beta=K / N$. In the following Proposition 2, we present the large-system results for the RCI-hybrid scheme.

Proposition 2: For the RCI-hybrid scheme, the largesystem secrecy sum rate and the large-system harvested power converge in probability to deterministic quantities given by (50) and (51) respectively, where $\phi=\alpha / N$ and $g(\beta, \phi)$ is the solution of $x$ to $x=\left(\phi+\frac{\beta}{1+x}\right)^{-1}$.

Proof: The results in Proposition 2 can be obtained using the procedure outlined in Theorem 1 and Proposition 1. Thus, the detailed proof is omitted here to avoid duplication.

\section{Optimal Design}

We now optimize the design of the RCI-hybrid scheme based on the large-system results. We determine the optimal regularization parameter $\alpha$, the optimal PS ratio $\rho$, and the optimal TS ratio $t$ that maximize the large-system secrecy sum rate subject to the energy-harvesting constraint.

Problem Formulation: The problem of optimizing the design of the RCI-hybrid scheme is formulated as:

$$
\begin{aligned}
\max _{\alpha, t, \rho} & R_{s, \text { hybrid }}^{\infty} \\
\text { s.t. } & E_{k, \text { hybrid }}^{\infty} \geq \Gamma, \alpha \geq 0,0 \leq t \leq 1,0 \leq \rho \leq 1 .
\end{aligned}
$$

Feasible Constraint: From (51), we find that the maximum achievable harvested power is obtained when $\alpha \rightarrow \infty, t \rightarrow 0$ and $\rho \rightarrow 0$. Thus, the feasible energy-harvesting constraint for the RCI-hybrid scheme is given by

$$
\Gamma<E_{k, \text { hybrid }}^{\infty}(\alpha \rightarrow \infty, t \rightarrow 0, \rho \rightarrow 0)=\epsilon \gamma P\left(1+\frac{1}{\beta}\right),
$$

which is the same as the feasible energy-harvesting constraint for the RCI-PS and RCI-TS schemes given in (21) and (43).
Optimal Solution: The optimal solution of $\alpha$, denoted by $\alpha_{\text {hybrid }}^{*}$, is given by

$$
\alpha_{\text {hybrid }}^{*}=f_{\alpha}\left(\rho=\rho_{\text {hybrid }}^{*}\right),
$$

where $\rho_{\text {hybrid }}^{*}$ denotes the optimal solution of $\rho$ and $f_{\alpha}(\rho)$ is defined in (23). The optimal solution of $t$, denoted by $t_{\text {hybrid }}^{*}$ and the optimal solution of $\rho$, denoted by $\rho_{\text {hybrid }}^{*}$, are obtained by numerically solving the problem:

$$
\begin{aligned}
\max _{t, \rho} & R_{s, \text { hybrid }}^{\infty}\left(t, \rho, \alpha=f_{\alpha}(\rho)\right), \\
\text { s.t. } & 1-\frac{\Gamma \beta}{\epsilon \gamma P(1+\beta)} \leq t \leq 1,0<\rho \leq \frac{1}{t}\left(1-\frac{\Gamma \beta}{\epsilon \gamma P(1+\beta)}\right) .
\end{aligned}
$$

In the following, we provide the proof of the proposed optimal solution for the RCI-hybrid scheme.

Proof: We can easily obtain $\alpha_{\text {hybrid }}^{*}$ as (55) based on the results for the RCI-PS scheme. We note that the closedform expressions for $t_{\text {hybrid }}^{*}$ and $\rho_{\text {hybrid }}^{*}$ are mathematically intractable. Hence, $t_{\text {hybrid }}^{*}$ and $\rho_{\text {hybrid }}^{*}$ are obtained by numerically solving the problem of (56). In addition, we find that the energy-harvesting constraint can be always achieved when $t \leq 1-\frac{\Gamma \beta}{\epsilon \gamma P(1+\beta)}$ for any given $\rho$ or $\alpha$. For a given $t \geq 1-\frac{\Gamma \beta}{\epsilon \gamma P(1+\beta)}$, the energy-harvesting constraint cannot be achieved if $\rho>\frac{1}{t}\left(1-\frac{\Gamma \beta}{\epsilon \gamma P(1+\beta)}\right)$. Thus, the ranges to search of $t$ and $\rho$ are given by (57).

\section{Numerical Results}

We first examine the accuracy of the large-system results. Table 3 illustrates the accuracy of the large-system approximations over the size of network for the RCI-hybrid scheme, where $\Delta R_{s}$ and $\Delta E_{k}$ are defined by (46) and (47), respectively. We set $\rho=0.8, t=0.8$, and $\alpha=0.2$. We note that both $\Delta R_{s}$ and $\Delta E_{k}$ decrease as $N$ increases. This indicates that the large-system approximations become more accurate as the size of network increases. As shown in the table, both $\Delta R_{s}$ and $\Delta E_{k}$ are smaller than $1 \%$ when $N \geq 24$. Thus, the large-system approximations for the RCI-hybrid scheme are very accurate even if the network size is limited.

We then compare the secrecy-energy tradeoffs achieved by the RCI-PS scheme, the RCI-TS scheme, and the RCI-hybrid scheme. Figure 7 plots $R_{s}^{\infty}$ versus $E_{k}^{\infty}$ for all three schemes. We find that the RCI-hybrid scheme always outperforms either the RCI-PS scheme or the RCI-TS scheme. Of course, the performance advantage of the RCI-hybrid scheme over the other two schemes is brought at the cost of the complexity in the receiver architecture with both a signal splitter and a switcher. It is worth mentioning that some literature pointed out that the TS sometimes can be regarded as a special case of the PS when the PS ratio can be changed anytime during the communication. However, the PS ratio is assumed to be constant over time in this work with the practical consideration of the limited capability of conventional splitters [31]. It has been shown in the literature, e.g., [30], that if the PS ratio is constant over time, the TS-based scheme sometimes outperforms the PS-based scheme and the PS-based scheme sometimes outperforms the TS-based scheme. Similarly, in 


$$
R_{s, \text { hybrid }}^{\infty}= \begin{cases}K t\left[\log _{2}\left(\frac{1+\frac{\rho \gamma P g(\beta, \phi)\left(1+\frac{\phi}{\beta}(1+g(\beta, \phi))^{2}\right)}{\rho \gamma P+\left(\rho \sigma_{n}^{2}+\sigma_{z}^{2}\right)(1+g(\beta, \phi))^{2}}}{1+\frac{\rho}{\left(\rho \sigma_{n}^{2}+\sigma_{z}^{2}(1)(1+g(\beta, \phi))^{2}\right.}}\right)\right]^{+}, & \text {if } \alpha>0 \\ K t \log _{2}\left(1+\frac{\rho \gamma P(1-\beta)}{\left(\rho \sigma_{n}^{2}+\sigma_{z}^{2}\right) \beta}\right), & \text { if } \alpha=0 \text { and } \beta \leq 1 \\ K t\left[\log _{2}\left(\frac{\beta^{4}+\frac{\rho \gamma P \beta^{3}(\beta-1)}{\rho \sigma_{n}^{2}+\sigma_{z}^{2}}}{\left(\beta^{2}+\frac{\rho \gamma P(\beta-1)}{\rho \sigma_{n}^{2}+\sigma_{z}^{2}}\right)^{2}}\right)\right]^{+}, & \text {if } \alpha=0 \text { and } \beta>1 .\end{cases}
$$

$$
E_{k, \text { hybrid }}^{\infty}= \begin{cases}\frac{t \epsilon(1-\rho) \gamma P(\beta+\phi g(\beta, \phi)(1+g(\beta, \phi)))}{\beta(1+g(\beta, \phi))}+(1-t) \epsilon \gamma P\left(1+\frac{1}{\beta}\right), & \text { if } \alpha \neq 0 \\ t \epsilon(1-\rho) \gamma P \frac{1-\beta}{\beta}+(1-t) \epsilon \gamma P\left(1+\frac{1}{\beta}\right), & \text { if } \alpha=0 \text { and } \beta \leq 1 \\ t \epsilon(1-\rho) \gamma P \frac{\beta-1}{\beta}+(1-t) \epsilon \gamma P\left(1+\frac{1}{\beta}\right), & \text { if } \alpha=0 \text { and } \beta>1\end{cases}
$$

Table 3: Illustration of the accuracy of large-system approximations for RCI-hybrid scheme.

\begin{tabular}{|c||c|c|c|c|c|c|c|c|c|c|}
\hline$N$ & 8 & 12 & 16 & 20 & 24 & 28 & 32 & 36 & 40 & 44 \\
\hline$\Delta R_{s}$ & $15.4 \%$ & $6.5 \%$ & $3.2 \%$ & $1.7 \%$ & $0.98 \%$ & $0.65 \%$ & $0.44 \%$ & $0.29 \%$ & $0.16 \%$ & $0.09 \%$ \\
\hline$\Delta E_{k}$ & $3.2 \%$ & $1.3 \%$ & $0.96 \%$ & $0.65 \%$ & $0.20 \%$ & $0.07 \%$ & $0.06 \%$ & $0.05 \%$ & $0.05 \%$ & $0.02 \%$ \\
\hline
\end{tabular}

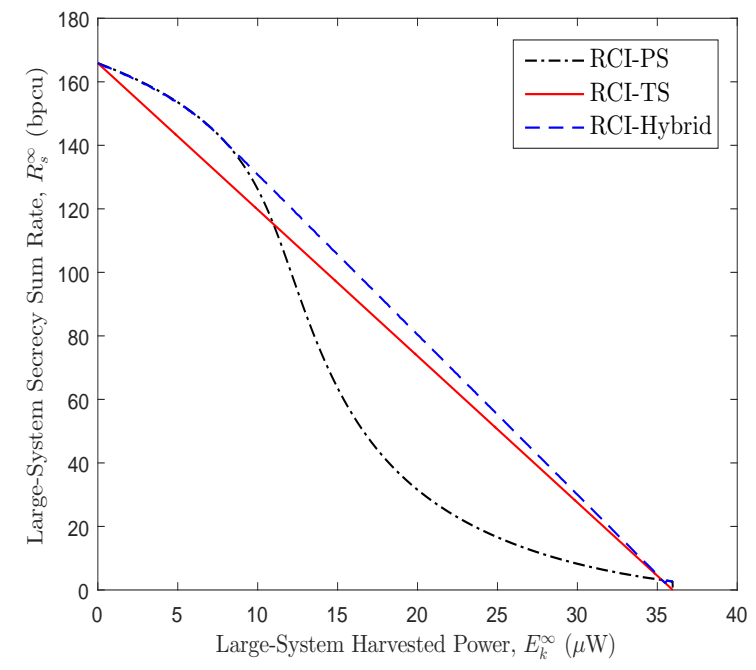

Fig. 7: The secrecy sum rate versus the harvested power.

this work, we find that the RCI-PS scheme sometimes outperforms the RCI-PS scheme and the RCI-TS scheme sometimes outperforms the RCI-PS scheme. Then, the proposed RCIhybrid scheme is practically significant since it combines both a signal splitter and a switcher, which leads to the fact that the RCI-hybrid scheme outperforms both the RCI-PS and RCI-TS schemes.

\section{CONCLUSion AND Future Work}

In this paper, we designed new transmission schemes to achieve SCBPT in a multiuser MISO network, based on the RCI precoding with the PS, the TS, and the newly proposed hybrid receiver architectures. We derived large-system expressions for the secrecy sum rate and the harvested power achieved by each scheme. Based on these expressions, we optimized the design of each scheme by determining the optimal regularization parameter of the RCI, the PS ratio, and the TS ratio that maximize the secrecy sum rate subject to the energy-harvesting constraint. Our numerical results corroborated the derived expressions and demonstrated the tradeoff between secrecy sum rate and harvested power achieved by each scheme. We showed that the RCI-hybrid scheme always outperforms both the RCI-PS and RCI-TS schemes.

As an initial work comprehensively studying transmission schemes for SCBPT, this paper has left several potential future work directions. One important direction is to consider the transmission schemes with finite-alphabet and non-Gaussian inputs for SCBPT. In this work, we have assumed that all users are using the same type of receiver device, so that the PS ratio is identical for all users. We note that a general scenario is that users have different receiver devices with different PS ratios. The analysis of non-identical $\rho$ for the general scenario is an interesting future direction. Furthermore, investigating the feasibility of some other physical layer security techniques such as the artificial noise injection for SCBPT is another research direction.

\section{APPENDIX A \\ PROOF OF THEOREM 1}

In the large-system analysis with $K, N \rightarrow \infty$, the secrecy rate for any message $s_{k}$ converges to a same non-random function, which does not depend on the realization of each channel $\mathbf{h}_{k}$. The secrecy sum rate is approximated by

$$
R_{s, \mathrm{PS}}^{\infty}=K\left(R_{k}^{\infty}\right)=K\left[\log _{2} \frac{1+\mathrm{SINR}_{k}^{\infty}}{1+\mathrm{SINR}_{\tilde{k}}^{\infty}}\right]^{+}
$$

where $R_{k}^{\infty}$ denotes the large-system secrecy rate for each user $k, \operatorname{SINR}_{k}^{\infty}$ and $\operatorname{SINR}_{\tilde{k}}^{\infty}$ denote the large-system approximations of the SINRs at the intended user and the eavesdropper, respectively. Also, the large-system harvested power at any user $k$ converges to a same non-random function, which does not depend on the realization of each channel $\mathbf{h}_{k}$. 
By applying the matrix inversion lemma, we have

$$
\left(\mathbf{H}^{H} \mathbf{H}+\alpha \mathbf{I}_{N}\right)^{-1}=\frac{1}{N} \mathbf{Z}_{k} .
$$

where $\mathbf{Z}_{k}=\mathbf{O}_{k}-\left(\mathbf{O}_{k}\left(\frac{1}{N} \mathbf{h}_{k}^{H} \mathbf{h}_{k}\right) \mathbf{O}_{k, j}\right) /\left(1+\frac{1}{N} \mathbf{h}_{k} \mathbf{O}_{k} \mathbf{h}_{k}^{H}\right)$ and $\mathbf{O}_{k}=\left(\frac{1}{N} \mathbf{H}_{\tilde{k}}^{H} \mathbf{H}_{\tilde{k}}+\frac{\alpha}{N} \mathbf{I}_{N}\right)^{-1}$.

We then rewrite the received SINRs at the information decoders of intended the user $k$ and the eavesdropper $\tilde{k}$ as $\operatorname{SINR}_{k}=\frac{\rho \gamma c^{2}\left|A_{k} /\left(1+A_{k}\right)\right|^{2}}{\rho \gamma c^{2} B_{k}+\rho \sigma_{n}^{2}+\sigma_{z}^{2}}$ and $\operatorname{SINR}_{\tilde{k}}=$ $\frac{\rho \gamma c^{2} B_{k}}{\rho \sigma_{n}^{2}+\sigma_{z}^{2}}$, respectively, where $A_{k}=\frac{1}{N} \mathbf{h}_{k} \mathbf{O}_{k} \mathbf{h}_{k}^{H}$ and $B_{k}=$ $\frac{1}{N} \mathbf{h}_{k} \mathbf{Z}_{k}\left(\frac{1}{N} \mathbf{H}_{\tilde{k}}^{H} \mathbf{H}_{\tilde{k}}\right) \mathbf{Z}_{k} \mathbf{h}_{k}^{H}$. In addition, we rewrite the harvested power at the energy harvester of user $k$ as $E_{k}=$ $\epsilon(1-\rho) \gamma c^{2}\left(\left|\frac{A_{k}}{1+A_{k}}\right|^{2}+B_{k}\right)$. Aided by [28,36], we obtain $A_{k} \stackrel{i . p .}{\longrightarrow} g(\beta, \phi), B_{k} \stackrel{i . p .}{\longrightarrow} \frac{1}{(1+g(\beta, \phi))^{2}}\left(g(\beta, \phi)+\phi \frac{\partial g(\beta, \phi)}{\partial \phi}\right)$, and $c^{2} \stackrel{a . s .}{\longrightarrow} \frac{P}{g(\beta, \phi)+\phi \frac{\partial g(\beta, \phi)}{\partial \phi}}$. In addition, we find that $g(\beta, \phi)+\phi \frac{\partial g(\beta, \phi)}{\partial \phi}=\frac{\beta g(\beta, \phi)}{\beta+\phi(1+g(\beta, \phi))^{2}}$. Applying these approximations, we obtain $\operatorname{SINR}_{k}^{\infty}=\frac{\rho \gamma P g(\beta, \phi)\left(1+\frac{\phi}{\beta}(1+g(\beta, \phi))^{2}\right)}{\rho \gamma P+\left(\rho \sigma_{n}^{2}+\sigma_{z}^{2}\right)(1+g(\beta, \phi))^{2}}$ and $\operatorname{SINR}_{\tilde{k}}^{\infty}=\frac{\rho \gamma P}{\left(\rho \sigma_{n}^{2}+\sigma_{z}^{2}\right)(1+g(\beta, \phi))^{2}}$. Then, we can easily get $R_{s, \mathrm{PS}}^{\infty}$ and $E_{k, \mathrm{PS}}^{\infty}$ for $\alpha \neq 0$ in (16) and (17), respectively. For the case of $\alpha=0$, we obtain $R_{s, \mathrm{PS}}^{\infty}$ and $E_{k, \mathrm{PS}}^{\infty}$ by calculating $R_{s, \mathrm{PS}}^{\infty}(\alpha=$ $0)=\lim _{\alpha \rightarrow 0} R_{s, \mathrm{PS}}^{\infty}$ and $E_{k, \mathrm{PS}}^{\infty}(\alpha=0)=\lim _{\alpha \rightarrow 0} E_{k, \mathrm{PS}}^{\infty}$, respectively. This completes the proof of Theorem 1 .

\section{APPENDIX B}

\section{PROOF OF COROLlaRY 1}

We note that proving $E_{k, \mathrm{PS}}^{\infty}$ is an increasing function of $\alpha$ is equivalent to proving $E_{k, \mathrm{PS}}^{\infty}$ is an increasing function of $\phi$, due to $\phi=\alpha / N$. As such, we examine if $f(\phi)=\partial E_{k, \mathrm{PS}}^{\infty} / \partial \phi$ is always positive for any $\phi>0$.

From (17), we have

$$
f(\phi)=\frac{4 \epsilon \gamma P(1-\rho) f_{1}(\phi)}{f_{2}(\phi)},
$$

where $f_{1}(\phi)=\left((1-\beta)^{2}+\beta \phi+\phi\right)-(\beta-1) \sqrt{4 \phi+(\beta+\phi-1)^{2}}$ and $f_{2}(\phi)=\sqrt{4 \phi+(\beta+\phi-1)^{2}}\left(\phi-\beta+\sqrt{4 \phi+(\beta+\phi-1)^{2}}+1\right)^{2}$. It is evident that $f_{2}(\phi)>0$ for any $\phi$. Thus, we have $\operatorname{sgn}\{f(\phi)\}=\operatorname{sgn}\left\{f_{1}(\phi)\right\}$. We find that $f_{1}(\phi)>0$ when $\beta \leq 1$. When $\beta<1$, we have $\operatorname{sgn}\left\{f_{1}(\phi)\right\}=\operatorname{sgn}\left\{4 \phi^{2} \beta\right\}>0$. Therefore, we confirm that $f(\phi)>0$ for any $\phi>0$. It follows that the maximum harvested power is obtained when $\alpha \rightarrow \infty$ given by (18). This completes the proof of Corollary 1.

\section{REFERENCES}

[1] P. Grover and A. Sahai, "Shannon meets Tesla: Wireless information and power transfer," in Proc. IEEE ISIT, Austin, TX, June 2010, pp. 2363-2367.

[2] X. Zhou, R. Zhang, and C. K. Ho, "Wireless information and power transfer: Architecture design and rate-energy tradeoff," IEEE Trans. Commun., vol. 61, no. 11, pp. 4754-4767, Nov. 2013.

[3] R. Zhang and C. K. Ho, "MIMO broadcasting for simultaneous wireless information and power transfer," IEEE Trans. Wireless Commun. vol. 12, no. 5, pp. 1989-2001, May 2013.

[4] L. Liu, R. Zhang, and K.-C. Chua, "Secrecy wireless information and power transfer with MISO beamforming," IEEE Trans. Signal Process., vol. 62, no. 7, pp. 1850-1863, Apr. 2014.
[5] D. W. K. Ng, E. S. Lo, and R. Schober, "Robust beamforming for secure communication in systems with wireless information and power transfer," IEEE Trans. Wireless Commun., vol. 13, no. 8, pp. 4599-4615, Aug. 2014.

[6] Q. Li, Q. Zhang, and J. Qin, "Secure relay beamforming for simultaneous wireless information and power transfer in nonregenerative relay networks," IEEE Trans. Veh. Technol., vol. 63, no. 5, pp. 2462-2467, Apr. 2014.

[7] H. Xing, K.-K. Wong, and A. Nallanathan, "Secure wireless energy harvesting-enabled af-relaying SWIPT networks," in IEEE ICC, June 2015, pp. 2307-2312.

[8] Z. Ding, I. Krikidis, B. Sharif, and H. V. Poor, "Wireless information and power transfer in cooperative networks with spatially random relays," IEEE Trans. Wireless Commun., vol. 13, no. 8, pp. 4440-4453, Aug. 2014.

[9] B. Zhu, J. Ge, Y. Huang, Y. Yang, and M. Lin, "Rank-two beamformed secure multicasting for wireless information and power transfer," IEEE Signal Process. Lett., vol. 21, no. 2, pp. 199-203, Feb. 2014.

[10] A. D. Wyner, "The wire-tap channel," Bell Syst. Tech. J., vol. 54, no. 8, pp. 1355-1387, Oct. 1975.

[11] M. Bloch and J. Barros, Physical-Layer Security: From Information Theory to Security Engineering. Cambridge University Press, 2011.

[12] X. Zhou, L. Song, and Y. Zhang, Physical Layer Security in Wireless Communications. CRC Press, 2013.

[13] N. Yang, L. Wang, G. Geraci, M. Elkashlan, J. Yuan, and M. Di Renzo, "Safeguarding 5G wireless communication networks using physical layer security," IEEE Commun. Mag., vol. 53, no. 4, pp. 20-27, Apr. 2015.

[14] R. Liu, T. Liu, H. V. Poor, and S. Shamai, "Multiple-input multipleoutput Gaussian broadcast channels with confidential messages," IEEE Trans. Inf. Theory, vol. 56, no. 9, pp. 4215-4227, Sept. 2010.

[15] D. A. A. Fakoorian and A. L. Swindlehurst, "MIMO interference channel with confidential messages: Achievable secrecy rates and precoder design," IEEE Trans. Inf. Forensics Security, vol. 6, no. 3, pp. 640-649, Sept. 2011.

[16] G. Geraci, M. Egan, J. Yuan, A. Razi, and I. B. Collings, "Secrecy sumrates for multi-user MIMO regularized channel inversion precoding," IEEE Trans. Commun., vol. 60, no. 11, pp. 3472-3482, Nov. 2012.

[17] G. Geraci, R. Couillet, J. Yuan, M. Debbah, and I. B. Collings, "Large system analysis of linear precoding in MISO broadcast channels with confidential messages," IEEE J. Sel. Areas Commun., vol. 31, no. 9, pp. 1660-1671, Sept. 2013.

[18] G. Geraci, A. Y. Al-Nahari, J. Yuan, and I. B. Collings, "Linear precoding for broadcast channels with confidential messages under transmit-side channel correlation," IEEE Commun. Lett., vol. 17, no. 6 , pp. 1164-1167, June 2013.

[19] N. Yang, G. Geraci, J. Yuan, and R. Malaney, "Confidential broadcasting via linear precoding in non-homogeneous MIMO multiuser networks," IEEE Trans. Commun., vol. 62, no. 7, pp. 2515-2530, July 2014.

[20] B. He, N. Yang, X. Zhou, and J. Yuan, "Base station cooperation for confidential broadcasting in multi-cell networks," IEEE Trans. Wireless Commun., vol. 14, no. 10, pp. 5287-5299, Oct. 2015.

[21] H. Zhang, C. Li, Y. Huang, and L. Yang, "Secure beamforming for SWIPT in multiuser MISO broadcast channel with confidential messages," IEEE Commun. Lett., vol. 19, no. 8, pp. 1347-1350, Aug. 2015.

[22] C. B. Peel, B. M. Hochwald, and A. L. Swindlehurst, "A vector-perturbation technique for near-capacity multiantenna multiuser communication-Part I: Channel inversion and regularization," IEEE Trans. Commun., vol. 53, no. 1, pp. 195-202, Jan. 2005.

[23] Y. Wu, C. Xiao, Z. Ding, X. Gao, and S. Jin, "Linear precoding for finitealphabet signaling over MIMOME wiretap channels," IEEE Trans. Veh. Technol., vol. 61, no. 6, pp. 2599-2612, July 2012.

[24] W. Dai, Y. Liu, B. C. Rider, and W. Gao, "How many users should be turned on in a multi-antenna broadcast channel?" IEEE J. Sel. Areas Commun., vol. 26, no. 8, pp. 1526-1535, Oct. 2008.

[25] H. Huh, A. M. Tulino, and G. Caire, "Network MIMO with linear zeroforcing beamforming: Large system analysis, impact of channel estimation, and reduced-complexity scheduling," IEEE Trans. Inf. Theory, vol. 58, no. 5, pp. 2911-2934, May 2012.

[26] R. Muharar, R. Zakhour, and J. Evans, "Optimal power allocation and user loading for multiuser MISO channels with regularized channel inversion," IEEE Trans. Commun., vol. 61, no. 12, pp. 5030-5041, Dec. 2013.

[27] L. Liu, R. Zhang, and K.-C. Chua, "Wireless information transfer with opportunistic energy harvesting," IEEE Trans. Wireless Commun., vol. 12 , no. 1 , pp. $288-300$, Jan. 2013. 
[28] R. Muharar, R. Zakhour, and J. Evans, "Base station cooperation with feedback optimization: A large system analysis," IEEE Trans. Inf. Theory, vol. 60, no. 6, pp. 3620-3644, June 2014.

[29] V. K. Nguyen and J. S. Evans, "Multiuser transmit beamforming via regularized channel inversion: A large system analysis," in Proc. IEEE GLOBECOM, New Orleans, LA, Nov. 2008, pp. 1-4.

[30] A. A. Nasir, X. Zhou, S. Durrani, and R. A. Kennedy, "Relaying protocols for wireless energy harvesting and information processing," IEEE Trans. Wireless Commun., vol. 12, no. 7, pp. 3622-3636, July 2013.

[31] M. Jalalifar and M. J. Uddin, "Power splitter architecture and applications," Progress in Electromagnetics Research C, vol. 18, pp. 231-244, 2011.

[32] A. Goldsmith, Wireless Communications. Cambridge University Press, 2005.

[33] S. Wagner, R. Couillet, M. Debbah, and D. T. M. Slock, "Large system analysis of linear precoding in correlated MISO broadcast channels under limited feedback," IEEE Trans. Inf. Theory, vol. 58, no. 7, pp. 4509-4537, July 2012.

[34] S. Yang and L. Hanzo, "Fifty years of MIMO detection: The road to large-scale mimos," IEEE Commun. Surv. Tut., vol. 17, no. 4, pp. 19411988, Fourthquarter 2015.

[35] A. Benjebbour, A. Harada, Y. Kishiyama, Y. Okumura, J. Ma, J. Qiu, D. Chen, L. Lu, and T. Kashima, "Large scale experimental trial of $5 \mathrm{G}$ air interface," in IEICE Gen. Conf., Sept. 2015, pp. B-5-63.

[36] R. Couillet and M. Debbah, Random Matrix Methods for Wireless Communications. Cambridge University Press, 2011.

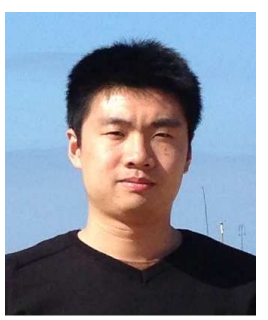

Biao He (M'16) received the B.E. (hons.) degree from the Australian National University (ANU) and Beijing Institute of Technology in 2012. He received the Ph.D. degree from the ANU in 2016. He is currently a Post-Doctoral Fellow at the Department of Electronic and Computer Engineering, The Hong Kong University of Science and Technology. His research interests include physical layer security and wireless communications.

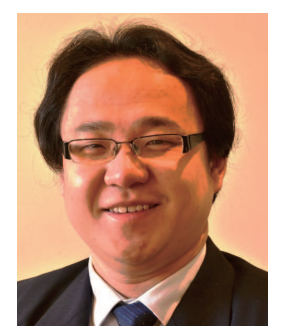

Nan Yang (S'09-M'11) received the Ph.D. degree in electronic engineering from the Beijing Institute of Technology in 2011. He is currently a Future Engineering Research Leadership Fellow and Lecturer in the Research School of Engineering at the Australian National University. Prior to this, he was a Postdoctoral Research Fellow with the University of New South Wales (2012-2014) and the Commonwealth Scientific and Industrial Research Organization (2010-2012). He received the Exemplary Reviewer Award of the IEEE Transactions on Communications and the Top Reviewer Award from the IEEE Transactions on Vehicular Technology in 2015, the IEEE ComSoc Asia-Pacific Outstanding Young Researcher Award and the Exemplary Reviewer Award of the IEEE Wireless Communications Letters in 2014, the Exemplary Reviewer Award of the IEEE Communications Letters in 2013 and 2012, and the Best Paper Award at the IEEE 77th Vehicular Technology Conference in 2013. He is currently serving in the Editorial Board of the IEEE Transactions on Vehicular Technology and the Transactions on Emerging Telecommunications Technologies. His general research interests include heterogeneous networks, massive multi-antenna systems, millimeter wave communications, cyberphysical security, and molecular communications.

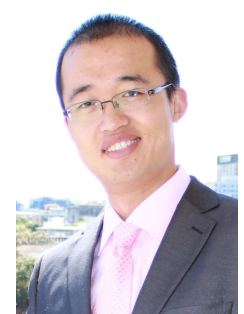

Shihao Yan (S'11-M'15) received the Ph.D degree in Electrical Engineering from The University of New South Wales, Sydney, Australia, in 2015. He received the B.S. in Communication Engineering and the M.S. in Communication and Information Systems from Shandong University, Jinan, China, in 2009 and 2012, respectively. He was a visiting Ph.D student at The University of South Australia, Adelaide, Australia, in 2014. He is currently a Postdoctoral Research Fellow in the Research School of Engineering, The Australia National University, Canberra, Australia. His current research interests are in the areas of wireless communications and statistical signal processing, including physical layer security, location verification, and localization algorithms.

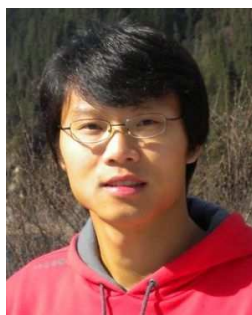

Xiangyun Zhou (M'11) received the Ph.D. degree in telecommunications engineering from the Australian National University (ANU) in 2010. From 2010 to 2011, he worked as a postdoctoral fellow at UNIK - University Graduate Center, University of Oslo, Norway. He returned to ANU in 2011 and currently works as a Senior Lecturer. His research interests are in the fields of communication theory and wireless networks. Dr. Zhou currently serves on the editorial board of IEEE TRANSACTIONS ON WIRELESS COMMUNICATIONS and IEEE COMMUNICATIONS LETTERS. He also served as a guest editor for IEEE COMMUNICATIONS MAGAZINE's feature topic on wireless physical layer security in 2015 and EURASIP Journal on Wireless Communications and Networking's special issue on energy harvesting wireless communications in 2014. He has also served as symposium/track/workshop co-chairs for major IEEE conferences. He was the chair of the ACT Chapter of the IEEE Communications Society and Signal Processing Society from 2013 to 2014. $\mathrm{He}$ is a recipient of the Best Paper Award at ICC'11. 